Mai 1993

\title{
T-PERIOD ECONOMIES \\ WITH INCOMPLETE MARKETS
}

\author{
Monique FLORENZANO * \\ Pascal GOURDEL **
}

$\mathrm{N}^{0} 9312$

* CNRS-CEPREMAP 140 rue du Chevaleret, 75013 Paris, France

** CERMSEM, Université de Paris I and Laboratoire d'Econométrie de l'Ecole Polytechnique, 1 rue Descartes, 75005 Paris, France 


\section{ECONOMIES A T PERIODES AVEC MARCHES INCOMPLETS}

\section{Resumé}

L'essentiel de la littérature sur les marchés incomplets concerne le cas simple d'un modèle à 2 périodes. L'existence de l'équilibre y est démontrée sous l'hypothèse d'actifs nominaux (rendements exprimés en unités de compte), d'actifs numéraires (rendements exprimés en unités d'un bien appelé numéraire) et d'actifs réels. Dans ce dernier cas, le résultat d'existence est seulement un résultat générique.

Dans un souci de réalisme mais aussi pour préparer un travail ultérieur sur les marchés incomplets en horizon infini, le résultat classique d'existence de l'équilibre en présence d'actifs nominaux est ici étendu à un modèle à plus de deux périodes.

Mots clés : Equilibre stochastique - Marchés incomplets - Actifs à rendements nominaux - Prix de non-arbitrage - Lemme de Debreu-Gale-Nikaido

\section{T-PERIOD ECONOMIES WITH INCOMPLETE MARKETS}

\footnotetext{
Abstract

The existence of stochastic equilibria is proved for a T-period economy with an incomplete financial stucture.

Key words : Stochastic equilibrium - Incomplete markets - Purely financial, one-period securities - No-arbitrage asset pricing - Debreu-Gale-Nikaido lemma.

JEL : D51, D52, D90
} 


\title{
T-period economies with incomplete markets
}

\author{
Monique Florenzano \\ CNRS-CEPRFMAP, 140 rue du Chevalenet, 75019 Paris, Fmnce,
}

Pascal Gourdel

CFRMSFM, Unizersité de Paris I and Labomtoire d'Fconométrie de l'Ecole Polytechnique, 1 rue Descartes, 75005 Paris, France.

A very simple proof is given for the existence of stochastic equilibria in a T-period economy with an incomplete financial structure.

Journal of Ficonomic litemtune, Classification Numbers D51, D52, D90.

\section{Introduction.}

In this paper, we investigate the existence of stochastic equilibria for a $T$-period economy with an incomplete financial structure.

Most of the literature on incomplete markets assumes a simple two-period model. In this framework, the existence of equilibrium was addressed by Cass (1984), Werner (1985 and 1989) in the case of nominal assets, Geanakoplos and Polemarchakis (1986) for real numeraire assets, Duffie and Shafer (1985) for real assets. In this last case, the existence result is only a generic result.

More recently, the literature has developed towards an analysis of economies with an open-ended future (see Hernandez and Santos (1991) and Magill and Quinzii (1992)). A generalization of equilibrium existence results from the two-period model to a sequential system of markets over a finite horizon is a necessary step for such an analysis : the equilibrium in an infinite horizon economy is generally viewed as a limit of equilibria of finite horizon economies. On the other hand, with its gradual resolution of uncertainty, a $T$-period economy is also a step in the direction of realism.

The $T$-period model was explored by Duffie (1987) for nominal assets and by Magill and Shafer (1991) for the case of real assets. Here, in order to get an existence result, we limit ourselves to the case of nominal assets. As it is standard in a model with nominal assets, we get that any security price process that precludes arbitrage can be embedded in an equilibrium.

Our other assumptions are quite general, essentially the same as in Duffie (1987). They could be weakened and have been choosen in order to prepare the ones to be used in the framework of an infinite horizon economy. Our proof is simple and follows the 
classical way of showing convenient continuity properties of an adapted excess-demand correspondence in order to apply the Debreu-Gale-Nikaido lemma to get the existence of equilibrium.

\section{The model.}

We consider an exchange economy with $(T+1)$ periods $t=0,1, \ldots, T$, and $m$ agents. The stochastic structure of the model is described by an event tree $S$ of the length $T$, with a unique initial node at date 0 and a finite branching number at each node of the tree. For every $t, S_{t}$ denotes the set of nodes occuring at date $t$ and $S^{t}$ the set of nodes before or during time period $t . S_{T}$ is the set of terminal nodes and $S=\bigcup_{t=0}^{T} S_{t}$. For any node $s \in S_{t}(t>0), s^{-}$denotes the (unique) node that immediately precedes $s$ at date $(t-1)$; for any node $s \in S_{t}(t<T), s^{+}$denotes the (finite) set of immediate successors of $s$.

At every node in the event tree $S$ described above, $L$ commodities are available and each consumer purchases commodities and trades securities on spot markets. On the commodity space $\mathbb{R}^{S \times L}$, each consumer is classically described by a consumption set $X^{i}=\mathbb{R}_{+}^{S \times L}$, a preference relation $\nwarrow^{i}$ on $X^{i}$ and a state-dependent initial endowment $\omega^{i} \in X^{i}$. On the other hand, at every non-terminal node $s$, agents participate in a financial market. We assume that the set $J_{s}$ of financial instruments available at $s$ is finite and that the only available financial assets are purely financial, one-period securities. Let $r^{j}\left(s^{\prime}\right)$ be the return in units of account that asset $j \in J_{s}$ promises to pay at $s^{\prime} \in s^{+}$and $r\left(s^{\prime}\right)=\left(r^{j}\left(s^{\prime}\right)\right)_{j \in J_{s}}$. The matrix of returns

$$
R_{s}=\left(r^{j}\left(s^{\prime}\right)\right)_{s^{\prime} \in s^{+}, j \in J_{s}}
$$

describes the financial structure at $s$ and $R=\prod_{s \in S^{T-1}} R_{s}$ denotes the financial structure of the economy. Finally, the economy is summarized by the list of data

$$
\mathcal{E}=\left(S, R,\left(X^{i}, \succsim^{i}, \omega^{i}\right)_{i=1}^{m}\right)
$$

At every $s$, consumers face a commodity price $p(s) \in \mathbb{R}^{L}$ and, if $s \in S^{T-1}$, an asset price $q(s) \in \mathbb{R}^{J_{s}}$. Their financial constraint is determined by the value at $p(s)$ of their initial endowment and if $t>0$ by the returns paid by their preceding portofolio. Let $z_{j}^{i}(s)$ denote the number of units of the $j^{\text {th }}$ security purchased by $i$ at $s$ (if $z_{j}^{i}(s)>0$ ) or sold (if $\left.z_{j}^{i}(s)<0\right)$ and $z^{i}(s)=\left(z_{j}^{i}(s)\right)_{j \in J_{s}} \cdot\left(x^{i}(s), z^{i}(s)\right)$ is the $i^{\text {th }}$ agent's consumption vector and portfolio at node $s$ and $\left.x^{i}=\left(x^{i}(s)\right)_{s \in S}, z^{i}=z^{i}(s)\right)_{s \in S^{T-1}}$ the consumption and portfolio 
plans of $i$ (note that consumers are required to pay back their debts in the final period). Taking a market system of commodity and asset price processes $(p, q)=(p(s), q(s))_{s \in S}$ as given, the budget set $B^{i}(p, q)$ is the set of $\left(x^{i}, z^{i}\right)$, such that $x^{i} \in X^{i}, z^{i} \in \prod_{s \in S^{T-1}} \mathbb{R}^{J_{s}}$ and

$$
\begin{gathered}
p(0) \cdot x^{i}(0)+\sum_{j \in J_{0}} q^{j}(0) z_{j}^{i}(0) \leq p(0) \cdot \omega^{i}(0) \\
p(s) \cdot x^{i}(s)+\sum_{j \in J_{s}} q^{j}(s) z_{j}^{i}(s) \leq p(s) \cdot \omega^{i}(s)+r(s) \cdot z^{i}\left(s^{-}\right) \forall s \in S^{T-1}, s \neq 0 \\
p(s) \cdot x^{i}(s) \leq p(s) \cdot \omega^{i}(s)+r(s) \cdot z^{i}\left(s^{-}\right) \forall s \in S_{T} .
\end{gathered}
$$

A budget feasible plan $\left(x^{i}, z^{i}\right)$ is optimal for $i$ if there is no other budget feasible plan $\left(x^{\prime i}, z^{\prime i}\right)$ for agent $i$ such that $x^{\prime i} \succ^{i} x^{i}$. The collection $\left((p, q),\left(x^{i}, z^{i}\right)_{i=1}^{m}\right)$ is an equilibrium if and only if

i) $\left(x^{i}, z^{i}\right)$ is an optimal budget feasible plan for each agent $i$ given $(p, q)$
ii) $\sum_{i=1}^{m} x^{i}=\sum_{i=1}^{m} \omega^{i}$
iii) $\sum_{i=1}^{m} z^{i}=0$

\section{T-period normalized nominal system}

If the agents are to have a solution to their consumption-portfolio choice problem, then the security price process must not offer arbitrage opportunities. A security price process $q$ is said arbitrage-free for $R$ if there is no trading strategy $z=(z(s)) \in \prod_{s \in S^{T-1}} \mathbb{R}^{J_{s}}$ such that $\Phi(z)>0$ with $\Phi(z) \stackrel{\text { def }}{=}\left\{\begin{array}{c}-q(0) \cdot z(0) \\ r(s) \cdot z\left(s^{-}\right)-q(s) \cdot z(s) \forall s \in S^{T-1}, s \neq 0 \\ r(s) \cdot z\left(s^{-}\right) \quad \forall s \in S_{T} .\end{array}\right.$

As in the two period model, we can invoke the strict separation theorem to characterize the no-arbitrage condition as a linear relationship between security prices and returns.

Lemma 1. If the market system $(R, q)$ does not permit any arbitrage, there exists $\lambda=$ $\left(\lambda_{s}\right)_{s \in S} \gg 0$ such that $\lambda_{0}=1$ and $\forall s \in S^{T-1} \lambda_{s} q(s)=\sum_{s^{\prime} \in s^{+}} \lambda_{s^{\prime}} r\left(s^{\prime}\right)$.

Proof. Let us denote by $\Delta_{S}$ the unit simplex

$$
\Delta_{S}=\left\{w=\left(w_{s}\right)_{s \in S} \mid \forall s, w_{s} \geq 0 \text { and }\|w\|_{1}=1\right\}
$$


It follows from the no arbitrage condition that $\operatorname{Im} \Phi \cap \Delta_{S}=\emptyset$. Since $\Delta_{S}$ is compact, we can apply the strict separation theorem to get $\beta \in \mathbb{R}^{S} \backslash\{0\}$ and $\alpha \in \mathbb{R}$ such that

$$
\sup _{x \in \operatorname{Im} \Phi} \beta \cdot x \leq \alpha<\inf _{y \in \Delta} \beta \cdot y
$$

Since the linear functional is majorized on the linear space $\operatorname{Im} \Phi$, it is identically equal to 0 on $\operatorname{Im} \Phi$. Then $\alpha \geq 0$ and it follows from the right inequality that $\beta_{s}>0 \forall s \in S$. If we denote by $\lambda$ the vector $\frac{\beta_{s}}{\beta_{0}}$, it verifies the conclusion since $\forall z \in \prod_{s \in S^{T-1}} \mathbb{R}^{J_{s}}$,

$$
\begin{aligned}
& \lambda \cdot \Phi(z)=-q(0) \cdot z(0)+\sum_{s \in S^{T-1} \backslash\{0\}} \lambda_{s}\left(r(s) \cdot z\left(s_{-}\right)-q(s) \cdot z(s)\right)+\sum_{s \in S_{T}} \lambda_{s} r(s) \cdot z\left(s^{-}\right) \\
& =-\sum_{s \in S^{T-1}} \lambda_{s} q(s) \cdot z(s)+\sum_{s \in T \backslash\{0\}} \lambda_{s} r(s) \cdot z\left(s^{-}\right) \\
& =-\sum_{s \in S^{T-1}} \lambda_{s} q(s) \cdot z(s)+\sum_{s \in S^{T-1}} \sum_{s^{\prime} \in s^{+}} \lambda_{s^{\prime}} r\left(s^{\prime}\right) \cdot z(s) \\
& =\sum_{s \in S^{T-1}}\left(\lambda_{s} q(s)-\sum_{s^{\prime} \in s^{+}} \lambda_{s^{\prime}} r\left(s^{\prime}\right)\right) \cdot z(s)=0 \\
& \Leftrightarrow \forall s \in S^{T-1}, \lambda_{s} q(s)=\sum_{s^{\prime} \in s^{+}} \lambda_{s^{\prime}} r\left(s^{\prime}\right) .
\end{aligned}
$$

$\lambda=\left(\lambda_{s}\right)_{s \in S}$ may be interpreted as a vector of strictly positive node (present value) prices ; indeed, if we mutiply by $\lambda_{s}$ each inequality defining the budget set $B^{i}(p, q)$ and if we sum, we get for any $x^{i}$ such that $\left(x^{i}, z^{i}\right) \in B^{i}(p, q), \pi \cdot x^{i} \leq \pi \cdot \omega^{i}$ with $\pi=\left(\lambda_{s} p(s)\right)_{s \in S}$.

Markets are said complete when the last inequality implies the existence of some portfolio plan $z^{i}$ such that $\left(x^{i}, z^{i}\right) \in B^{i}(p, q)$. Using the standard notation

$$
p \square\left(x^{i}-\omega^{i}\right)=\left(p(s) \cdot\left(x^{i}(s)-\omega^{i}(s)\right)_{s \in S}\right.
$$

markets are said complete when $\pi \cdot x^{i} \leq \pi \cdot \omega^{i} \Rightarrow p \square\left(x^{i}-\omega^{i}\right) \in \operatorname{Im} \Phi$. In this case, it is equivalent for each consumer to optimize in the budget set $B^{i}(p, q)$ previously defined or in the Arrow-Debreu budget set $B^{i}(p, q, \lambda)=\left\{x^{i} \in X^{i} \mid \pi \cdot x^{i} \leq \pi \cdot \omega^{i}\right\}$.

Lemma 2. Markets are complete if and only if in Lemma 1, $\lambda$ is unique.

Proof. Assume $\lambda$ is unique. Then $\operatorname{dim}\left((\operatorname{Im} \Phi)^{\perp}\right)=1$. As $p \square\left(x^{i}-\omega^{i}\right)=u+v$ with $u \in \operatorname{Im} \Phi$ and $v=\alpha \lambda$ for some $\alpha \in \mathbb{R}, 0=\lambda \cdot\left(p \square\left(x^{i}-\omega^{i}\right)\right)=\lambda \cdot u+\lambda \cdot v=\alpha\|\lambda\|^{2} \Rightarrow \alpha=0$ and $v=0$. Assume now that $\lambda \cdot\left(p \square\left(x^{i}-\omega^{i}\right)\right)=0 \Rightarrow p \square\left(x^{i}-\omega^{i}\right) \in \operatorname{Im} \Phi$. Then if $[\lambda]=\mathbb{R} \lambda,[\lambda]^{\perp} \subset \operatorname{Im} \Phi$ and $(\operatorname{Im} \Phi)^{\perp} \subset[\lambda]$. This shows that $\operatorname{dim}\left((\operatorname{Im} \Phi)^{\perp}\right)=1$ and $\lambda$ is unique. 
When markets are incomplete, the multiplicity of the inequalities defining the budget constraint matters. In this case, as observed by Magill and Quinzi (1992), at an equilibrium $\left(\bar{p}, q,\left(\bar{x}^{i}, \bar{z}^{i}\right)\right)$, each consumer $i$ has a (possibly different) system $\lambda^{i}=\left(\lambda_{s}^{i}\right)_{s \in S}$ of (present value) node price satisfying the conclusions of Lemma 1 , such that $\bar{x}^{i}$ optimizes its preferences in its Arrow-Debreu budget constraint $B^{i}\left(\bar{p}, q, \lambda^{i}\right)$.

In the following, $\lambda=\left(\lambda_{s}\right)_{s \in S}$ of wich the existence was proved in Lemma 1 will be used for a normalization of prices.

\section{Existence Theorem}

We will prove the existence of an equilibrium under the following assumptions :

$A$ 1. For $i=1, \ldots, m$, the relation $\succsim^{i}$ is a complete, convex, monotone and continuous preorder satisfying $y \succ^{i} x$ and $\alpha \in\left[01\left[\Rightarrow \alpha x+(1-\alpha) y \succ^{i} x\right.\right.$.

$A$ 2. For each $i$, in each state of nature, there is a direction which is extremely desirable. Formally $\forall i, \forall s \in S, \exists \chi_{s}^{i} \in \mathbb{R}^{L \times S}$ such that $\forall s^{\prime} \neq s, \chi_{s}^{i}\left(s^{\prime}\right)=0$ and for all $x^{i} \in X^{i}$, $x^{i}+\chi_{s}^{i} \succ^{i} x^{i}$

$A$ 3. For $i=1, \ldots, m, \omega^{i} \gg 0$.

We also assume that the price process $q$ is arbitrage free for $R$.

Let us denote by $\Delta$ the unit simplex of the price processes.

$$
\Delta=\left\{p=(p(s))_{s \in S} \in \mathbb{R}^{L \times S} \mid \forall s, p(s) \geq 0 \text { and }\|p\|_{1}=1\right\} .
$$

In view of Assumption A2, we will look at equilibrium prices in $P=\left\{p=(p(s))_{s \in S} \in\right.$ $\left.\mathbb{R}^{L \times S} \mid \forall s, p(s)>0\right\}$.

Lemma 3. There exists a closed set $F \subset \mathbb{R}^{S}$, such that $B^{i}(p, q)=\left\{x^{i} \in X^{i} \mid p \square\left(x^{i}-\omega^{i}\right) \in\right.$ $F\}$. Hence the correspondences $B^{i}$ are convex valued on $\Delta$ and closed.

Proof. With a slight abuse of notation, let us write $B^{i}(p, q)=\left\{x^{i} \in X^{i} \mid \exists z^{i}\right.$ such that $\left.p \square\left(x^{i}-\omega^{i}\right) \leq \Phi\left(z^{i}\right)\right\}=\left\{x^{i} \in X^{i} \mid p \square\left(x^{i}-\omega^{i}\right) \in \operatorname{Im} \Phi-\mathbb{R}_{+}^{S}\right\}$. It follows from the no-arbitrage condition that the two cones $\operatorname{Im} \Phi$ and $-\mathbb{R}_{+}^{S}$ are positively semi-independant. Hence, we deduce that the set $F=\operatorname{Im} \Phi-\mathbb{R}_{+}^{S}$ is closed, (cf. Debreu 1959, chapter 1). Clearly by linearity of the box product, the correspondences $B^{i}$ are convex valued and closed. 
Using a trick due to Cass (1984), we allow now the first consumer to optimize his (her) preferences on the larger set

$$
B^{1}(p, q, \lambda)=\left\{x^{1} \in X^{1} \mid \sum_{s \in S} \lambda_{s} p(s) \cdot\left(x^{1}(s)-\omega^{1}(s)\right) \leq 0\right\}
$$

By definition of $\lambda$, we have $B^{1}(p, q) \subset B^{1}(p, q, \lambda)$.

Lemma 4. The correspondences $p \rightarrow B^{1}(p, q, \lambda)$ and $p \rightarrow B^{i}(p, q), i=2, \ldots, m$, are lower semicontinuous on $P \cap \Delta$.

Proof. For the first consumer, the result is classical, so we will restrict our attention to $i=2, \ldots, m$. First, let us remark that, for all $p \in P \cap \Delta, p \square \omega^{i} \gg 0$ for all $i$. If we take $x^{i} \in B^{i}(p, q)$, we have for some $z^{i}, p \square\left(x^{i}-\omega^{i}\right) \leq \Phi\left(z^{i}\right)$. Hence, we deduce for all $\mu \in\left[0,1\left[, p \square\left(\mu x^{i}-\omega^{i}\right) \leq \Phi\left(\mu z^{i}\right)\right.\right.$. This proves that $B^{i}(p, q)=\operatorname{cl}\left(\delta^{i}(p, q)\right)$ where

$$
\delta^{i}(p, q)=\left\{x^{i} \in X^{i} \mid \exists z^{i} \text { such that } p \square\left(x^{i}-\omega^{i}\right) \ll \Phi\left(z^{i}\right)\right\} .
$$

This implies that $B^{i}(p, q)$ is lower semicontinuous at $p$ as closure of an open graph correspondence.

We can now define as follows a truncated economy. $\hat{B}^{i}(p, q)$ is the truncated budget set $B^{i}(p, q) \cap[0,2 \omega]$ with $\omega=\sum_{i=1}^{m} \omega^{i}$. The truncated demand correspondences $\hat{\xi}^{i}$ are defined by

$$
\hat{\xi}^{i}(p)= \begin{cases}\left\{x^{1} \in \hat{B}^{1}(p, q, \lambda) \text { such that }\left\{x^{\prime} \in \hat{B}^{1}(p, q, \lambda) \mid x^{\prime} \succ x^{1}\right\}=\emptyset\right\}, & i=1 \\ \left\{x^{1} \in \hat{B}^{i}(p, q) \text { such that }\left\{x^{\prime} \in \hat{B}^{i}(p, q) \mid x^{\prime} \succ x^{i}\right\}=\emptyset\right\}, & i \neq 1\end{cases}
$$

Using the normalization of prices defined in section 3 , we will use the following notations $\pi=(\pi(s)) \stackrel{\text { déf }}{=}\left(\lambda_{s} p(s)\right)$ and $\hat{\xi}^{i}(\pi) \stackrel{\text { déf }}{=} \hat{\xi}^{i}(p)$. On the other hand, for each (big enough) integer $\nu$, let us define $\Delta_{\nu}$ by

$$
\Delta_{\nu}=\left\{p=(p(s))_{s \in S} \in \mathbb{R}^{L \times S} \mid \forall s, \forall k \in\{1, \ldots, L\}, p_{k}(s) \geq \frac{1}{\nu} \text { and }\|p\|_{1}=1\right\} .
$$

It follows from the construction of the $\hat{\xi}^{i}$, Lemmas 3 and 4 and the continuity of preferences, that each correspondence $\pi \rightarrow \hat{\xi}^{i}(\pi)$ is upper semicontinuous on $\Delta_{\nu}$ with nonempty convex values. Moreover, using the construction of $\lambda$, we obtain that for all $\pi \in \Delta_{\nu}$, for all $x^{i} \in \hat{\xi}^{i}(\pi), \pi \cdot\left(x^{i}-\omega^{i}\right) \leq 0$. Let us define the excess-demand correspondence $\zeta$ by $\zeta(\pi)=\sum_{i=1}^{m}\left(\hat{\xi}^{i}(\pi)-\omega^{i}\right)$. 
We can apply the Debreu-Gale-Nikaido lemma to the correspondence $\zeta$ to get the existence of $\left(Z^{\nu}, \pi^{\nu}\right)$ such that $\pi^{\nu} \in \Delta_{\nu}, Z^{\nu} \in \zeta\left(\pi^{\nu}\right)$ and for all $\pi \in \Delta_{\nu}, \pi \cdot Z^{\nu} \leq \pi^{\nu} \cdot Z^{\nu} \leq 0$.

By definition of $\zeta$, for all $i=1, \ldots, m$, there exists $x^{i, \nu} \in \hat{\xi}^{i}\left(\pi^{\nu}\right)$ such that $Z^{\nu}=$ $\sum_{i=1}^{m}\left(x^{i, \nu}-\omega^{i}\right)$. Without loss of generality, we may assume that the sequence $\left(\left(x^{i, \nu}\right), \pi^{\nu}\right)$ is convergent, say $\left(\left(x^{i, \nu}\right), \pi^{\nu}\right) \rightarrow\left(\left(\bar{x}^{i}\right), \bar{\pi}\right)$.

Lemma 5. The allocation $\left(\bar{x}^{i}\right)_{i=1}^{m}$ is feasible.

Proof. Taking limits as $\nu \rightarrow+\infty$, we obtain for all $\pi \in \Delta$ and $\pi \gg 0, \pi \cdot\left(\sum_{i=1}^{m} \bar{x}^{i}-\sum_{i=1}^{m} \omega^{i}\right) \leq 0$. Thus, we must have $\sum_{i=1}^{m} \bar{x}^{i} \leq \sum_{i=1}^{m} \omega^{i}$.

Lemma 6. For all $s \in S, \bar{\pi}(s) \neq 0$ and for all $i=1, \ldots, m, \bar{x}^{i} \in \hat{\xi}^{i}(\bar{\pi})$.

Proof. Assume on the contrary that there exists $s$ such that $\bar{\pi}(s)=0$. From the assumption 3 , we have $\bar{\pi} \cdot \omega^{1}>0=\inf \bar{\pi} \cdot X^{1}$, which implies that the budget correspondence of the first consumer is lower semicontinuous at $\bar{\pi}$ and that $\bar{x}^{1} \in \hat{\xi}^{1}(\bar{\pi})$. From Lemma 4 , we have that $\bar{x}^{1} \leq \omega$. But there exists $\mu>0$ such that $\bar{x}^{1}+\mu \chi_{s}^{1} \leq 2 \omega$ and by Assumption 2, $\bar{x}^{1}+\mu \chi_{s}^{1} \succ \bar{x}^{1}$. Since $\bar{\pi} \cdot \chi_{s}^{1}=0$, there is a contradiction. Finally, for $i=2, \ldots, m$ since $\bar{\pi} \in P$, we have from Lemma 4 that the correspondences $\hat{\xi}^{i}$ are upper semicontinuous at $\bar{\pi}$.

Theorem 1. Under the assumptions 1,2 and 3 , and if the price process $q$ is arbitrage-free for $R$, then the economy $\mathcal{E}$ has an equilibrium $\left((\bar{p}, q),\left(\bar{x}^{i}, \bar{z}^{i}\right)_{i=1}^{m}\right)$ with $\bar{p} \in P \cap \Delta$.

Proof. By classical arguments, it follows from the convexity assumption made in A1 on preferences that for all $i=1, \ldots, m, \bar{x}^{i} \in \xi^{i}(\bar{\pi})$. For $i=2, \ldots, m$, let $\bar{z}^{i}$ be the portfolio associated with $\bar{x}^{i}$. Let us remark that the local non-satiation assumed by A2 implies that $\bar{\pi} \cdot\left(\bar{x}^{1}-\omega^{1}\right)=0$ and $\bar{p} \square\left(x^{i}-\omega^{i}\right)=\Phi\left(\bar{z}^{i}\right)$ for all $i=2, \ldots, m$. From this, it follows that $\bar{\pi} \cdot\left(\bar{x}^{i}-\omega^{i}\right)=0$ for all $i$. Hence, we can replace $\bar{x}^{1}$ by $\omega-\sum_{i=2}^{m} \bar{x}^{i}$ which is in the budget constraint of the first consumer and by monotonicity of its preferences at least as good for him as $\bar{x}^{1}$.

We can now construct the portfolio of the first consumer by : $\bar{z}^{1}=-\sum_{i=2}^{m} \bar{z}^{i}$. Since 
for all $i=2, \ldots, m, \bar{p} \square \bar{x}^{i}=\Phi\left(\bar{z}^{i}\right)$, this implies that $\bar{p} \square \sum_{i=2}^{m} \bar{x}^{i}=\Phi\left(\sum_{i=2}^{m} \bar{z}^{i}\right)$ and finally $\bar{p} \square \bar{x}^{1}=\Phi\left(\bar{z}^{1}\right)$. Since $\left(\bar{x}^{1}, \bar{z}^{1}\right) \in B^{1}(\bar{p}, q)$, it is an optimal feasible plan for agent 1 .

Corollary. If the preferences of one consumer are strictly monotone, then the equilibrium price $\bar{p}$ is strictly positive.

\section{Proof. Obvious.}

The assumption on preferences in the corollary corresponds to the one made in Hernandez and Santos (1991).

\section{References}

Cass, D. (1984), Competitive equilibrium with financial incomplete markets, CARESS working paper no. 84-09 (University of Pennsylvania, Philadelphia, PA).

Debreu, G. (1959), Theory of Value, Wiley.

Duffie, D. (1987), Stochastic Equilibria with financial incomplete markets, Journal of Economic Theory, 41,405416.

Duffie, D. and W. Shafer (1985), Stochastic Equilibria with financial incomplete markets, Journal of Mathematical Economics, 14,285-300.

Hernandez, A. and M. Santos (1991), Incomplete Financial Market in Infinite Horizon Economies, Discussion paper, University of Wisconsin-Madison.

Geanakoplos, J. and H. Polemarchakis (1986), Existence, Regularity and constrained suboptimality of Competitive allocations when Markets are Incomplete, W.P. Heller, R.M. Ross and D.A. Starrett eds., Uncertainty, Information and Communication, Essays in Honor of Kenneth Arrow, Vol. 3, Cambridge University Press, Cambridge.

Magill, M. and M. Quinzi (1992), Infinite Horizon Incomplete Markets, Working paper.

Magill, M. and W. Shafer (1991), Incomplete Markets, Handbook of Mathematical Economics, Volume IV, North Holland, Amsterdam.

Werner, J. (1985), Equilibrium in economies with financial incomplete markets, Journal of Economic Theory, 36, 110-119.

Werner, J.(1989), Equilibrium with incomplete markets without ordered preferences, Journal of Economic Theory, 45, 379-382. 